\title{
Study of combination algorithm in partitioning manufacture technology
}

\author{
WANG Hui-feng, ZHANG Yong-jun, HAN Jin-tao, CHEN Ling \\ School of Material Science and Engineering, University of Science and Technology Beijing, Beijing \\ 100083
}

Keyword: Model Partitioning; Feature-based; Combination algorithm; BAAG

\begin{abstract}
Partitioning Manufacture Technology (PMT), which can get complex parts by simple processing has been developed to reduce tooling cost or increase machining efficiency. For the piece number is the primary reason influencing the machining efficiency, to reduce it, a combination algorithm is introduced based on the original feature-based partitioning algorithm. It consists of three steps: (1) Input a pieces set orderly. (2) Built a body attribute adjacency graph (BAAG) to describe the relationship of all pieces in the researching partitioning scheme; (3) Combine the vertices in BAAG, whose combination does not influence the visibility, to reduce the pieces number step by step until there is no redundancy partitioning (RP). At the end of paper, the partitioning schemes with and without combination algorithm are compared. From the comparison we can see introduction of combination algorithm can reduce the pieces number observably. It shows that the improved algorithm can fit to industry better then the former.
\end{abstract}

\section{Introduction}

When a complex product or model can't be machined as a whole it needs to be partitioned into a number of smaller and simpler manufacturable pieces. After manufactured, the individual pieces can be assembled to get the original product or model. This technology has been used widely in industry, such as prototype production, stock generation and mold design ${ }^{[1-3]}$. To reduce human factors in partitioning results, increase the precision and efficiency of computation, and ensure the rationality of partitioning scheme, a feature-based partitioning algorithm for complex model has been presented in our previous paper ${ }^{[4]}$.

In this paper, to reduce the pieces number of partitioning, a combination algorithm is introduced in the previous algorithm. Section 2 describes the process of the previous algorithm; Section 3 describes the realization of combination algorithm; Section 4 describes the comparison of partitioning results with and without combination algorithm; Section5 gives the conclusions.

\section{Related works}

There are many published papers on part partitioning for layered manufacturing, it is a single direction partitioning, such as $\operatorname{Hur}^{[3-5]}$, Ramaswami et al ${ }^{[6]}$ etc.

The single direction partitioning simply the process of partitioning, but it will bring more piece number synchronously. So, multi-directions partitioning is studied. Ki ${ }^{[7]}$ described a part decomposition algorithm for die pattern machining, where different partitioning directions are allowed for each layer so that the minimum number of layers can be obtained. But it did not touch on the problem of selection of optimal partitioning schemes. Dhaliwal et al. ${ }^{[2,8]}$ described some decomposition methods for the design of multi-piece mold. This method can achieve multi-direction partitioning. However, the facets modeling results in a large computation and low efficiency. 
A feature-based partitioning algorithm is studied in our previous paper. It takes features as the investigated subjects to increase computational efficiency and precision and uses multi-direction partitioning to reduce piece number. Fig.1 shows the procedure of feature-based partitioning algorithm. It includes four steps: features recognition, visibility analysis, model partitioning and optimal scheme selection.

\section{Step1: Features recognition}

In this step, Graph-based feature recognition ${ }^{[9]}$ is used to achieve feature recognition. It contains three main steps, B-rep model extraction, Attributed Adjacency Gragh (AAG) construction and Sub-AAGs extraction. In our algorithm some typical features are defined and the feature matching helps to get the machining features in the part.

\section{Step2: Visibility analysis}

In this step, visibility analysis is used for machinability analysis under some hypothesis. Local visibility ${ }^{[10]}$ and global visibility analysis are usedto judge whether the model or features could be machined using3-axis CNC machining, in turn.

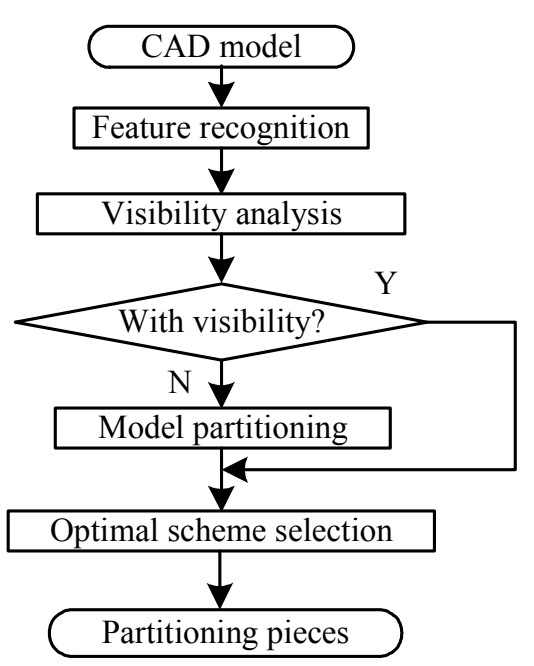

Step3: Model partitioning

Candidate Faces $(\mathrm{Fc})$ are defined in this step firstly, and then, Fig.1 Flowchart of the feature-based to solve some problems, which affect the material utilization and model partitioning machining precision, two restrictions are introduced in the algorithm.

\section{Step4: Optimal scheme selection}

The pieces number is taken as the primary objective to choose the optimal scheme from all candidate partitioning schemes. If two partition schemes have same pieces number the other three secondary will be introduced in turn and a selection rule will be considered simultaneously.

In partitioning process, the partitioning face split the model into two parts and may produce the Redundancy Partitioning (RP). RP is defined: if there are at least two pieces, in the machining pieces set after model partition, can be combined under the machinability condition, the partitioning scheme has RP. Introduction of combination algorithm can reduce the number of partitioning pieces obviously. It will be inserting after step3 and before step4.

\section{Combination algorithm}

In this section, a mold design case is studied to illustrate the combination process. Fig.2a shows the part. Fig.2b shows the gross mold of part ${ }^{[11]}$. Fig.2c shows one of the partitioning schemes using the feature-based partitioning algorithm introduced in the above section. The partitioning scheme is the subject investigated.

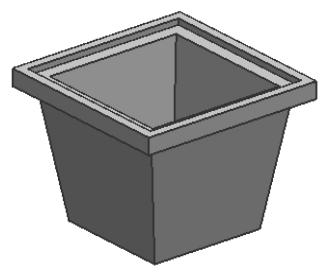

a) A part

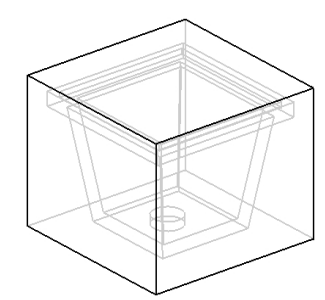

b) Gross mold of part

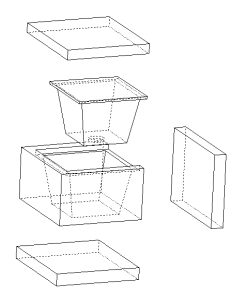

c) One partitioning scheme

Fig.2 Partitioning model and one partitioning scheme 


\subsection{Description of problem}

Suppose $\mathrm{P}_{\mathrm{S}}$ as one pieces set of model partitioning scheme and $P_{1} \in P_{S}, P_{2} \in P_{S}, P_{1} \neq P_{2}$. When the $\operatorname{UNITE}\left(P_{1}, P_{2}\right)$, the union of $\mathrm{P}_{1}$ and $\mathrm{P}_{2}$, can not get a connected model, it is $\operatorname{UNITE}\left(P_{1}, P_{2}\right)=\operatorname{NULL}$. Otherwise, $\operatorname{UNITE}\left(P_{1}, P_{2}\right) \neq N U L L \quad$.When $\operatorname{UNITE}\left(P_{1}, P_{2}\right) \neq N U L L$ and $\exists\left(P_{1}, P_{2}\right), \operatorname{Vis}\left(\operatorname{UNTEE}\left(P_{1}, P_{2}\right)\right)=1$, it is $R P\left(P_{S}\right)=1$, otherwise $R P\left(P_{S}\right)=0$.

If $R P\left(P_{S}\right)=1$, there are redundancy partitioning in the scheme and need combinations. The combination problem of partitioning pieces can be summarized as following: Input: the set of partitioning pieces with visibility after partitioning, $P_{S}$. Output: the set of partitioning pieces with visibility after combination, $P_{S}^{\prime}$. Constraint conditions of output: $R P\left(P_{S}^{\prime}\right)=0$

\subsection{Combination strategy of partitioning pieces}

Combination algorithm can be achieved in three steps:

1) Input a $P_{S}$ orderly. Fig.2c shows a $P_{S}$ including 5 pieces.

2) A body attribute adjacency graph (BAAG) is built to describe the relationship of piece bodies. The attribute between two adjacency bodies record whether the two have visibility after they are combined. An adjacency list is used to memorize the BAAG.

3) Traverse all bodies in BAAG and carry out the following operations.

\subsection{Case of study}

Taking the case shown in Fig. $2 \mathrm{c}$ as an example, from the BAAG in the left of Fig.3a we can see the relationships of all piece bodies in the partitioning scheme. Fig.3b shows the result of combination $\mathrm{P}_{4}$ andP $\mathrm{P}_{5}$. One piece body is reduced after that. $\mathrm{P}_{3}$ andP $\mathrm{P}_{45}$ are combined in Fig. $3 \mathrm{c}$ and there is only one attribute signed 1 in the BAAG. After combination of $\mathrm{P}_{1}$ and $\mathrm{P}_{2}$, there are only two vertices in the BAAG and the final partitioning pieces are $\mathrm{P}_{12}$ and $\mathrm{P}_{345}$, as shown in Fig. $3 \mathrm{~d}$. The numbers of pieces before and after combination algorithm are 5 and 2 respectively. It shows that the combination algorithm can reduce piece number obviously.

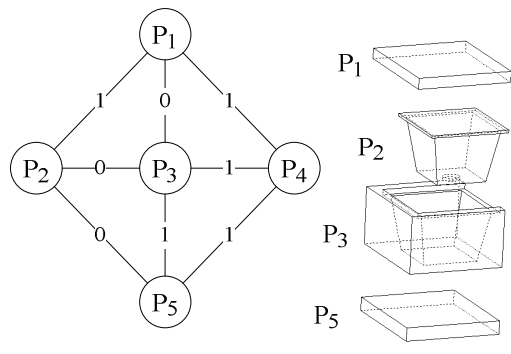

(a)

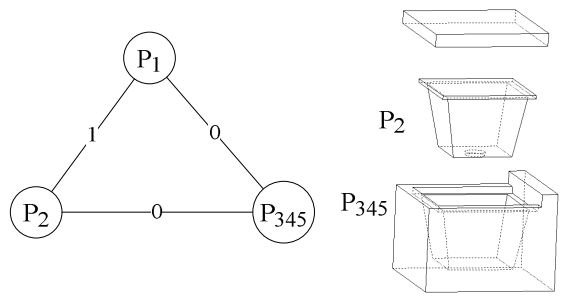

(c)

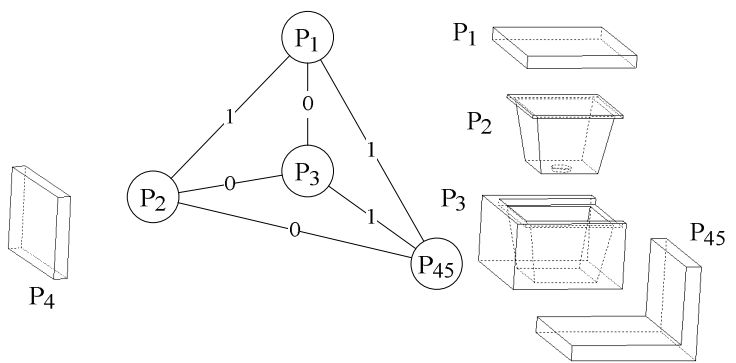

(b)
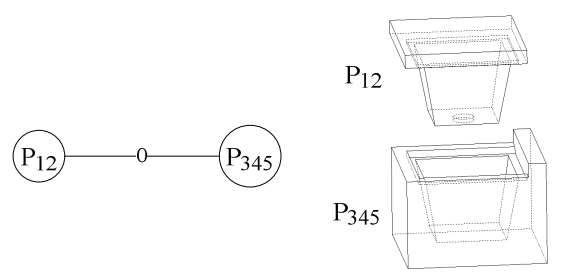

(d)

Fig.3 Case study of combination process 


\section{Comparisons}

Combination algorithm in the feature-based partitioning (before step4), can get less pieces number. In this section, a part (shown in Fig.4) is studied used two algorithms. Table1 shows the partitioning schemes of feature-based partitioning algorithm and Table 2 shows the results after introducing combination algorithm. In table1 the maximum number is 6 and the minimum is 3 and in table 2 the maximum is 3 and the minimum is 2 . It can be seen that the number of partitioning pieces reduced obviously in the Table2. Fig.6 and Fig.7 show the optimal schemes gotten from the two algorithms respectively. There are 3 pieces in Fig.5.

Corresponding numbers of machining features is 4,

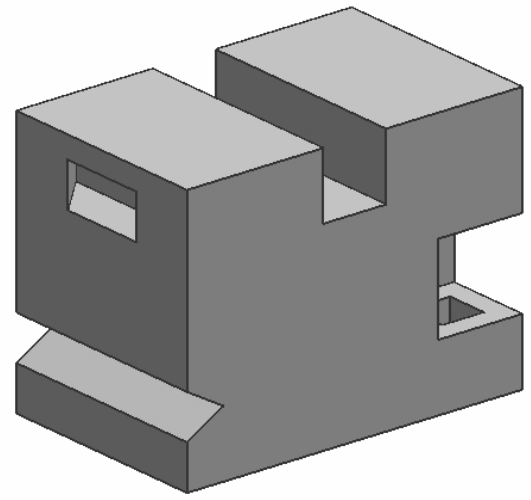

Fig.4 The studied part partitioning area is 0.00394954 and material utilization is 0.857911 . There are 2 pieces in Fig.6. Corresponding numbers of machining features is 6, partitioning area is 0.00395 and material utilization is 0.75522 . From the comparison, we can see although the material utilization increase in the improved algorithm, the other two elements are both reduced obviously. So the introduction of combination algorithm is better than the origin one in industry production.

Table1 The partitioning schemes of feature-based partitioning algorithm

\begin{tabular}{|c|c|c|c|c|}
\hline $\begin{array}{c}\text { Serial } \\
\text { number }\end{array}$ & $\begin{array}{c}\text { Pieces } \\
\text { numbers }\end{array}$ & $\begin{array}{c}\text { Numbers of } \\
\text { machining features }\end{array}$ & $\begin{array}{c}\text { Partitioning } \\
\text { area }\end{array}$ & $\begin{array}{c}\text { Material } \\
\text { utilization }\end{array}$ \\
\hline 1 & 3 & 5 & 0.00625 & 0.894104 \\
\hline 2 & 5 & 3 & 0.00761699 & 0.864441 \\
\hline 3 & 6 & 4 & 0.00525 & 0.94635 \\
\hline 4 & 3 & 4 & 0.00394954 & 0.857911 \\
\hline 5 & 4 & 7 & 0.0057 & 0.894104 \\
\hline 6 & 4 & 5 & 0.00655 & 0.908296 \\
\hline 7 & 3 & 7 & 0.00357013 & 0.846489 \\
\hline 8 & 4 & 4 & 0.00655 & 0.938076 \\
\hline
\end{tabular}

Table2 The results after combination algorithm introduced.

\begin{tabular}{|c|c|c|c|c|}
\hline $\begin{array}{c}\text { Serial } \\
\text { number }\end{array}$ & $\begin{array}{c}\text { Pieces } \\
\text { numbers }\end{array}$ & $\begin{array}{c}\text { Numbers of } \\
\text { machining features }\end{array}$ & $\begin{array}{c}\text { Partitioning } \\
\text { area }\end{array}$ & $\begin{array}{c}\text { Material } \\
\text { utilization }\end{array}$ \\
\hline 1 & 3 & 6 & 0.0051 & 0.894104 \\
\hline 2 & 2 & 9 & 0.0039317 & 0.894104 \\
\hline 3 & 2 & 6 & 0.00395 & 0.75522 \\
\hline 4 & 3 & 7 & 0.003825 & 0.819415 \\
\hline 5 & 3 & 8 & 0.0033067 & 0.752928 \\
\hline 6 & 3 & 6 & 0.00395 & 0.800316 \\
\hline 7 & 3 & 7 & 0.00357013 & 0.846489 \\
\hline 8 & 3 & 5 & 0.004925 & 0.811667 \\
\hline 9 & 3 & 5 & 0.00445 & 0.809753 \\
\hline 10 & 3 & 6 & 0.0038 & 0.81359 \\
\hline
\end{tabular}




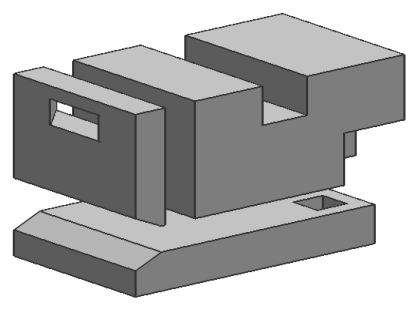

Fig. 5 The result of previous algorithm

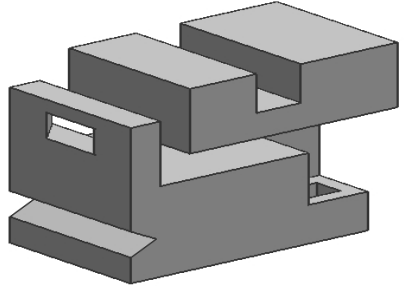

Fig.6 The result of improved algorithm

\section{Conclusions}

In this paper, a combination algorithm is studied based on the previous feature-based partitioning algorithm to increase machining efficiency. This algorithm can ensure the minimal pieces number in the partitioning schemes. The advantage of improved partitioning algorithm is proved through the comparison of the partitioning results.

Feature technology is combined with model partition and features are taken as study primitives. In partitioning process, numbers of machining features are used as one of the subsidiary objective to get optimal algorithm. Number of machining features can influence the clamp frequency directly and determine the machining efficiency. Feature-based visibility analysis can get global visibility more efficiency and accurately than existing methods. Most existing algorithms used triangle facets modeling as objective. Large amount of triangle facets will result in low computational efficiency and for triangle facets modeling is an approximate model, it will cause low precision.

Since this approach automatically produces solid models of model components, it can be integrated with CAM systems to generate the cutter path plans for manufacturing the individual components.

\section{References}

1. Hayong Shin, Gustav J. Olling, Yun C. Chung, Bo H. Kim and SuK. Cho, An integrated CAPP/CAM system for stamping die pattern machining, Computer-Aided Design, 2003, 35: 203-213.

2. Dhaliwal S, Gupta SK, Huang J, Kumar M. A feature-based approach to automated design of multi-piece sacrificial molds. ASME J Comput Inform Sci Engng 2001, 1(3):225-34.

3. J.H. Hur, K.W. Lee, H. Zhu, J.W. Kim, Hybrid rapid-prototyping system using machining and deposition [J]. Computer-Aided Design, 2002, 34 (10):741-754.

4. WANG Hui-feng, ZHOU Xiong-hui, WANG Wan, The Feature-based Partitioning Algorithm for Complex Part[J]. Journal of Shanghai Jiaotong University .2007, 41(9) : 1492-1496.

5. Zhu Hu_, Kunwoo Lee, Concave edge-based part decomposition for hybrid rapid prototyping, International Journal of Machine Tools \& Manufacture, 2005, 45: 35 - 42.

6. Y.C. Chang, J.M. Pinilla, J.H. Kao, J. Dong, K. Ramaswami, F.B. Prinz, Automated layer decomposition for additive/subtractive solid freeform fabrication, Proceedings of the Solid Freeform Fabrication Symposium, The University of Texas at Austin, August 1999.

7. D.W. Ki, K.W. Lee, Part decomposition for die pattern machining, Journal of Materials Processing Technology , 2002, 130-131:599-607.

8. J. Huang, S.K. Gupta, K. Stoppel,Generating sacrificial multi-piece molds using accessibility driven spatial partitioning,Computer-Aided Design, 2003, 35:1147-1160.

9. S. Joshi, T.C. Chang. Graph-Based Heuristics for Recognition of Machined Features from a 3D Solid Model. Computer-Aided Design. 1988, 20(2):58-66.

10. Woo T C. Visibility maps and spherical algorithms. Computer Aided Design, 1994, 26(1): 6-16.

11. Dhaliwal S, Gupta SK, Huang J, Kumar M. A feature-based approach to automated design of multi-piece sacrificial molds. ASME J Comput Inform Sci Engng 2001, 1(3):225-34. 